\title{
Efficient estimation for the heteroscedastic single-index varying coefficient models
}

\author{
Peng Lai ${ }^{a}$, Qingzhao Zhang ${ }^{b, *},{\text { Heng } \operatorname{Lian}^{c} \text { and Qihua Wang }}^{d}$ \\ ${ }^{a}$ School of Mathematics and Statistics, Nanjing University of Information Science $\mathcal{E}$ Technology \\ ${ }^{b}$ School of Economics and the Wang Yanan Institute for Studies in Economics, Xiamen University \\ ${ }^{*}$ Corresponding author \\ ${ }^{c}$ School of Mathematics and Statistics, University of New South Vales \\ ${ }^{d}$ Academy of Mathematics and Systems Science, Chinese Academy of Science
}

\begin{abstract}
We propose a semiparametric estimator for the heteroscedastic single-index varying coefficient model. The estimator is proved to attain the semiparametric efficiency bound. Some simulation studies and a real data application are conducted to evaluate the proposed methods.
\end{abstract}

Keywords: Single-index varying coefficient model; efficiency; semiparametric efficiency bound; estimating equation.

\section{Introduction}

The varying coefficient model has gained a lot of attentions during the past decade. Compared to the parametric model, it is more flexible to capture the true underlying relationships between covariates and response. However, the varying coefficient model

$$
Y=\alpha(U)^{\top} Z+\varepsilon, \quad Z \in R^{q}
$$

would suffer from the "curse of dimensionality" problem if the dimension of $U$ is high. This difficulty motivates us to study more flexible and more general models. We consider a heteroscedastic single-index varying coefficient model of the form

$$
Y=g^{\top}\left(X^{\top} \beta\right) Z+\varepsilon
$$


where $X \in R^{p}$ and $Z \in R^{q}$ are vectors of covariates, $Y$ is the response variable, $\beta$ is a $p \times 1$ vector of unknown parameters with $\|\beta\|=1$ for the sake of identifiability $(\|\cdot\|$ denotes the Euclidean metric), $g(\cdot)$ is a $q \times 1$ vector of unknown functions and $\varepsilon$ is a random error with $E(\varepsilon \mid X, Z)=0$ and $\operatorname{Var}(\varepsilon \mid X, Z)=\sigma^{2}(X, Z), \sigma^{2}(X, Z)$ is some unknown function.

Model (2) includes many important statistical models as special cases. If $g(\cdot)$ is a $q \times 1$ constant vector, it turns to the linear regression model. If $p=1$ and $\beta=1$, it reduces to the varying coefficient model. If some elements of $g(X)$ are constants, it is the varying coefficient partially linear model. If $q=1$ and $Z=1$, it reduces to the single-index model. And if $p=q$ and $Z=X$, model (2) becomes the adaptive varying coefficient linear model. Thus, model (2) is easily utilized in real applications since it is flexible enough to capture many different situations.

Existing literature focused on homoscedastic variance case with $\sigma^{2}(X, Z)$ being a positive constant. Xue and Wang (2012) developed an adjusted empirical likelihood ratio approach for constructing the confidence regions of the parameter vector $\beta$. Huang and Zhang (2013) proposed a maximum empirical likelihood estimator. Feng and Xue (2013, 2015) studied some variable selection problems in the single-index varying coefficient model. To the best of our knowledge, there are no existing studies on the heteroscedastic situation, which is very common in practice.

Moreover, the aforementioned papers did not consider semiparametric efficiency problem. Among a class of regular asymptotically linear estimators, semiparametric efficient estimators, which achieve the so-called semiparametric efficiency bound, have the smallest asymptotic variance. Tsiatis (2006) gave a general introduction to semiparametric efficiency bound and efficient estimators. Semiparametric efficiency problems have been studied extensively. Ma and Zhu (2013) studied the efficient estimators for the heteroscedastic partially linear single-index models allowing high dimensional covariates by constructing a semiparametrically efficient score function. Xue and Pang (2013) studied the statistical properties for the parametric estimator of (2) with homoscedastic variance, and further studied its efficiency when model (2) reduced to the single-index model. Lai and Wang (2014) and Lai et al. (2014) studied the efficient estimators for the partially linear single-index models. 
In this paper, our goal is to search for the efficient estimator of $\beta$ for the heteroscedastic single-index varying coefficient model (2). The rest of this paper is organized as follows. In Section 2, the semiparametric efficient estimator for the parameter vector $\beta$ is defined and the asymptotic properties of the proposed estimators are also investigated. In Section 3, numerical studies are reported. All technical details are delayed to Appendix.

\section{Semiparametric efficient estimator}

In order to obtain the efficient estimator for $\beta$ in model (2), we firstly define the semiparametrically efficient score function for the parameter of interest $\beta$, which can be used to construct the efficient estimating equations.

Suppose that $\left\{\left(Y_{i}, X_{i}, Z_{i}\right), i=1, \ldots, n\right\}$ is a random sample generated from the singleindex varying coefficient model (2). For the sake of identifiability, we assume that $\|\beta\|=1$ and the first component of $\beta$ is positive. This assumption means that the true value of $\beta$ is a boundary point on the unit sphere, and hence $g\left(X^{\top} \beta\right)$ does not have a derivative at the point $\beta$ (Zhu and Xue (2006)). For the convenience of computing the score vector, we use the delete-one-component method proposed by Yu and Ruppert (2002). Let $\beta=$ $\left(\beta_{1}, \ldots, \beta_{p}\right)^{\top}$ and $\beta^{(1)}=\left(\beta_{2}, \ldots, \beta_{p}\right)^{\top}$, then, we may write

$$
\beta\left(\beta^{(1)}\right)=\left(\left(1-\left\|\beta^{(1)}\right\|^{2}\right)^{1 / 2}, \beta_{2}, \ldots, \beta_{p}\right)^{\top} .
$$

The true parameter vector $\beta_{0}^{(1)}$ must satisfy the constraint $\left\|\beta_{0}^{(1)}\right\|<1$. Thus, $\beta$ is infinitely differentiable in a neighborhood of $\beta_{0}^{(1)}$, and the Jacobian matrix is

$$
J_{\beta^{(1)}}=\frac{\partial \beta}{\partial \beta^{(1)}}=\left(b_{1}, \ldots, b_{p}\right)^{\top}
$$

where $b_{s}(1<s \leq p)$ is a $(p-1)$-dimensional unit vector with $s$ th component 1 , and $b_{1}=$ $-\left(1-\left\|\beta^{(1)}\right\|^{2}\right)^{-1 / 2} \beta^{(1)}$. Thus, we can compute the efficient score function $S_{\beta}^{\text {eff }}(Y, X, Z)$ for the heteroscedastic single-index varying coefficient model in the following theorem.

Theorem 1 Assume that the conditional probability density function of $\varepsilon$ given $(X, Z)$, $p_{1}(\varepsilon \mid X, Z)$, is differentiable with respect to $\varepsilon$ and $0<E\left(\varepsilon^{2} \mid X, Z\right)<\infty$ almost everywhere. Then the semiparametrically efficient score of model (2) is given by

$$
\begin{aligned}
S_{\beta_{0}}^{e f f}(Y, X, Z)= & \varepsilon w(X, Z)\left\{g^{\prime \top}\left(X^{\top} \beta_{0}\right) Z J_{\beta_{0}^{(1)}}^{\top} X-E\left[w(X, Z) g^{\prime \top}\left(X^{\top} \beta_{0}\right) Z\right.\right. \\
& \left.\left.\times J_{\beta_{0}^{(1)}}^{\top} X Z^{\top} \mid X^{\top} \beta_{0}\right]\left\{E\left[w(X, Z) Z Z^{\top} \mid X^{\top} \beta_{0}\right]\right\}^{-1} Z\right\},
\end{aligned}
$$


where $\varepsilon=Y-Z^{\top} g\left(X^{\top} \beta_{0}\right)$ and $w(X, Z)=\left\{E\left(\varepsilon^{2} \mid X, Z\right)\right\}^{-1}$.

This theorem provides us the semiparametrically efficient score function for $\beta$ even for the heteroscedastic model when $w(X, Z)=\left\{E\left(\varepsilon^{2} \mid X, Z\right)\right\}^{-1}=\left\{\sigma^{2}(X, Z)\right\}^{-1}$. By Theorem 4.1 of Tsiatis (2006), it is proved directly that the semiparametric efficiency bound of model (2) is

$$
\left\{E\left(S_{\beta_{0}}^{e f f} S_{\beta_{0}}^{e f f^{\top}}\right)\right\}^{-1}=\left\{E\left(w(X, Z) S_{e}\left(\beta_{0}\right) S_{e}^{\top}\left(\beta_{0}\right)\right)\right\}^{-1}
$$

where

$$
\begin{aligned}
S_{e}\left(\beta_{0}\right) & =g^{\prime \top}\left(X^{\top} \beta_{0}\right) Z J_{\beta_{0}^{(1)}}^{\top} X-D_{2}\left(X^{\top} \beta_{0}\right) D_{1}^{-1}\left(X^{\top} \beta_{0}\right) Z, \\
D_{1}\left(X^{\top} \beta_{0}\right) & =E\left[w(X, Z) Z Z^{\top} \mid X^{\top} \beta_{0}\right], \\
D_{2}\left(X^{\top} \beta_{0}\right) & =E\left[w(X, Z) g^{\prime \top}\left(X^{\top} \beta_{0}\right) Z J_{\beta_{0}^{(1)}}^{\top} X Z^{\top} \mid X^{\top} \beta_{0}\right] .
\end{aligned}
$$

Based on Theorem 1, the following efficient estimating equation

$$
\sum_{i=1}^{n} \hat{S}_{\beta}^{e f f}\left(Y_{i}, X_{i}, Z_{i}\right)=0
$$

can be constructed to compute the semiparametrically efficient estimator whose asymptotic variance attains the semiparametric efficiency bound, where

$$
\begin{aligned}
\hat{S}_{\beta}^{e f f}\left(Y_{i}, X_{i}, Z_{i}\right)= & \left\{Y_{i}-Z_{i}^{\top} \hat{g}\left(X_{i}^{\top} \beta\right)\right\} \hat{w}\left(X_{i}, Z_{i}\right)\left\{\hat{g}^{\prime \top}\left(X_{i}^{\top} \beta\right) Z_{i} J_{\beta^{(1)}}^{\top} X_{i}\right. \\
& \left.-\hat{E}\left[\hat{w}\left(X_{i}, Z_{i}\right) \hat{g}^{\prime \top}\left(X_{i}^{\top} \beta\right) Z_{i} J_{\beta^{(1)}}^{\top} X_{i} Z_{i}^{\top} \mid X_{i}^{\top} \beta\right]\left\{\hat{E}\left[\hat{w}\left(X_{i}, Z_{i}\right) Z_{i} Z_{i}^{\top} \mid X_{i}^{\top} \beta\right]\right\}^{-1} Z_{i}\right\},
\end{aligned}
$$

with $\hat{g}(\cdot), \hat{g}^{\prime}(\cdot), \hat{E}\left(\cdot \mid X^{\top} \beta\right)$ and $\hat{w}(\cdot)$ denoting the consistent estimators of $g(\cdot), g^{\prime}(\cdot), E\left(\cdot \mid X^{\top} \beta\right)$ and $w(\cdot)$ respectively. Such estimators can be obtained as follows.

For a given $\beta$, we employ the local linear regression technique (Fan and Gijbels (1996)) to estimate $g(\cdot)$ and $g^{\prime}(\cdot)$. The local linear estimators for $g(t)$ and $g^{\prime}(t)$ are defined as $\hat{g}(t)=\hat{a}$ and $\hat{g}^{\prime}(t)=\hat{b}$, where $\hat{a}$ and $\hat{b}$ minimize the sum of weighted squares

$$
\sum_{i=1}^{n}\left[Y_{i}-\left\{a+h b \frac{X_{i}^{\top} \beta-t}{h}\right\}^{\top} Z_{i}\right]^{2} K_{h}\left(X_{i}^{\top} \beta-t\right),
$$

with respect to $a$ and $b, K_{h}(\cdot)=\frac{1}{h} K(\cdot / h), K(\cdot)$ is a kernel function and $h$ is a bandwidth. Therefore, we can obtain

$$
\left(\begin{array}{c}
\hat{g}(t) \\
h \hat{g}^{\prime}(t)
\end{array}\right)=S_{n}^{-1}(t, \beta) \xi_{n}(t, \beta),
$$


where

$$
S_{n}(t, \beta)=\left(\begin{array}{cc}
S_{n, 0}(t, \beta) & S_{n, 1}(t, \beta) \\
S_{n, 1}(t, \beta) & S_{n, 2}(t, \beta)
\end{array}\right), \quad \xi_{n}(t, \beta)=\left(\begin{array}{c}
\xi_{n, 0}(t, \beta) \\
\xi_{n, 1}(t, \beta)
\end{array}\right)
$$

and

$$
\begin{aligned}
S_{n, k}(t, \beta) & =\frac{1}{n} \sum_{i=1}^{n} Z_{i} Z_{i}^{\top}\left(\frac{X_{i}^{\top} \beta-t}{h}\right)^{k} K_{h}\left(X_{i}^{\top} \beta-t\right), k=0,1,2 ; \\
\xi_{n, k}(t, \beta) & =\frac{1}{n} \sum_{i=1}^{n} Z_{i} Y_{i}\left(\frac{X_{i}^{\top} \beta-t}{h}\right)^{k} K_{h}\left(X_{i}^{\top} \beta-t\right), k=0,1 .
\end{aligned}
$$

To obtain the nonparametric estimators of the conditional expectations in (5), the NadarayaWatson kernel method is used here. We define the estimators respectively by

$$
\begin{aligned}
& \hat{E}\left[\hat{w}(X, Z) \hat{g}^{\prime \top}\left(X^{\top} \beta\right) Z J_{\beta^{(1)}}^{\top} X Z^{\top} \mid X^{\top} \beta\right] \\
& =\frac{\sum_{i=1}^{n} K_{h}\left(X_{i}^{\top} \beta-X^{\top} \beta\right) \hat{w}\left(X_{i}, Z_{i}\right) \hat{g}^{\prime \top}\left(X_{i}^{\top} \beta\right) Z_{i} J_{\beta^{(1)}}^{\top} X_{i} Z_{i}^{\top}}{\sum_{i=1}^{n} K_{h}\left(X_{i}^{\top} \beta-X^{\top} \beta\right)} \\
& \hat{E}\left[\hat{w}(X, Z) Z Z^{\top} \mid X^{\top} \beta\right]=\frac{\sum_{i=1}^{n} K_{h}\left(X_{i}^{\top} \beta-X^{\top} \beta\right) \hat{w}\left(X_{i}, Z_{i}\right) Z_{i} Z_{i}^{\top}}{\sum_{i=1}^{n} K_{h}\left(X_{i}^{\top} \beta-X^{\top} \beta\right)} .
\end{aligned}
$$

Note that $w(X, Z)=\left\{E\left(\varepsilon^{2} \mid X, Z\right)\right\}^{-1}=\frac{1}{\sigma^{2}(X, Z)}$. Clearly, the nonparametric regression estimator of $\sigma^{2}(X, Z)$ suffers from the curse of dimensionality when the dimensionality of $(X, Z)$ is high. To overcome this difficulty, Ma and Zhu (2013) assume there is a low dimensional variable $\xi=\xi(X, Z)$ such that $\sigma^{2}(X, Z)=\operatorname{var}(\varepsilon \mid \xi)$ and $\xi$ has a known form and $\xi$ is univariate. In this paper, we assume that there exists a link function $\varphi(\cdot)$ and parameter $\gamma_{0}$ such that

$$
\sigma^{2}(X, Z)=\operatorname{var}(\varepsilon \mid X, Z)=\varphi\left(\gamma_{0}^{\top} U\right)
$$

where $U=\left(X^{\top}, Z^{\top}\right)^{\top}$ and $\left\|\gamma_{0}\right\|=1$. Hence, the single-index model is utilized to model the heterogeneity. Let $\hat{\varepsilon}_{i}=Y_{i}-Z_{i}^{\top} \hat{g}_{I}\left(X_{i}^{\top} \hat{\beta}_{I}\right)$, where $\hat{\beta}_{I}$ and $\hat{g}_{I}(\cdot)$ are the consistent initial estimators, which can be obtained by solving (5) and minimizing (6) with $w(X, Z) \equiv 1$. Thus, considering $E\left[\varepsilon_{i}^{2} \mid U\right]=\varphi\left(\gamma_{0}^{\top} U\right), \gamma_{0}$ and $\varphi(\cdot)$ can be estimated by the usual kernel method, for example Carroll et al. (1997), and we obtain $\hat{\sigma}^{2}(X, Z)$. Two bandwidths $h_{1}$ and $h_{2}$ are additionally involved. The bandwidth $h_{1}$ is selected for estimation of $\gamma_{0}$, while $h_{2}$ is for estimation of $\varphi(\cdot)$. These bandwidths $h, h_{1}$ and $h_{2}$ in the nonparametric estimating process can be selected by the cross-validation technique. 
Define the solution of $(5)$ on $\beta^{(1)}, \hat{\beta}^{(1)}$ say, to be the estimator of $\beta_{0}^{(1)}$. For $\beta \in \mathbf{B}_{n}$ in (5), $\mathbf{B}_{n}=\left\{\beta:\left\|\beta-\beta_{0}\right\| \leq C n^{-1 / 2}\right\}$, and a positive constant $C$, we have the following asymptotic properties.

Theorem 2 If model (2) is a heteroscedastic model with $E\left(\varepsilon^{2} \mid X, Z\right)=\sigma^{2}(X, Z)$, where $\sigma^{2}(X, Z)$ is twice times continuously differentiable with bounded derivative, then we have that, under the regularity conditions C1-C8 given in the Appendix,

$$
\sqrt{n}\left(\hat{\beta}^{(1)}-\beta_{0}^{(1)}\right) \stackrel{\mathcal{D}}{\longrightarrow} N\left(0, \Sigma_{1}^{-1}\right),
$$

where

$$
\Sigma_{1}=E\left[w(X, Z) S_{e}\left(\beta_{0}\right) S_{e}\left(\beta_{0}\right)^{\top}\right]
$$

Corollary 1 Under the conditions of Theorem 2, we have

$$
\sqrt{n}\left(\hat{\beta}-\beta_{0}\right) \stackrel{\mathcal{D}}{\longrightarrow} N\left(0, J_{\beta_{0}^{(1)}} \Sigma_{1}^{-1} J_{\beta_{0}^{(1)}}^{\top}\right) .
$$

Remark 1 From Theorem 2, we can see that the asymptotic variance of the estimator is $\Sigma_{1}^{-1}$, hence attains the semiparametric efficiency bound proposed in (4).

\section{Numerical examples}

\subsection{Simulations}

To illustrate the finite sample performances of the proposed method, we consider the example below. In the simulations, the Epanechnikov kernel $K(t)=0.75\left(1-t^{2}\right)_{+}$is used. Moreover, since root-n consistency of the proposed estimators does not require undersmoothing of nonparametric functions, the optimal bandwidth can be selected to be the optimal one for estimation of nonparametric functions. We use 5-fold cross validation to choose bandwidths for estimators.

Let $\left\{Y_{i}, X_{i}, Z_{i} ; i=1, \cdots, n\right\}$ be the i.i.d sample where $X_{i}=\left(X_{i 1}, \cdots, X_{i p}\right)^{\top}$ is generated from uniform distribution on $[0,1]^{p}$ with independent components, $Z_{i 1}=1$ and $\left(Z_{i 2}, \cdots, Z_{i q}\right)^{\top}$ follows a multivariate normal distribution with $\operatorname{cov}\left(Z_{i j_{1}}, Z_{i j_{2}}\right)=0.5^{\left|j_{1}-j_{2}\right|}$ for $2 \leq j_{1}, j_{2} \leq q$. Consider the model

$$
Y_{i}=\sum_{s=1}^{q} g_{s}\left(X_{i}^{\top} \beta\right) Z_{i(s+1)}+0.5 e_{i}
$$


where $\beta=\left(\beta_{1}, \cdots, \beta_{p}\right)^{\top}$ with true value $\beta_{0}$ and $e_{i}$ is the error term. We set $p=2, q=3$ and consider the model $(10)$ with $g_{1}(u)=2 \sin (2 \pi u), g_{2}(u)=\exp (2 u-1), g_{3}(u)=8 u(1-$ $u)$, and $\beta_{0}=(2,1)^{\top} / \sqrt{5}$. Two error distributions were considered: (1-1)homoscedastic: $e_{i} \sim N(0,1)$ and (1-2)heteroscedastic: $e_{i} \sim N\left(0, \exp \left(U_{i}^{\top} \gamma_{0} / 2\right)\right)$ with $U_{i}=\left(X_{i}^{\top}, Z_{i 2}, \cdots, Z_{i q}\right)^{\top}$ and $\gamma_{0}=(1, \cdots, 1) / \sqrt{p+q-1}$. Let $n=100$ and 200, and a total of 500 simulation replications were conducted.

To examine the performances of the efficient estimator, we consider the following four different estimating equations:

$$
\begin{aligned}
& \text { (a) } n^{-1} \sum_{i=1}^{n}\left\{Y_{i}-Z_{i}^{\top} \hat{g}\left(X_{i}^{\top} \beta\right)\right\} \hat{w}\left(X_{i}, Z_{i}\right)\left\{\hat{g}^{\top \top}\left(X_{i}^{\top} \beta\right) Z_{i} J_{\beta^{(1)}}^{\top} X_{i}\right. \\
& \left.-\hat{E}\left[\hat{w}\left(X_{i}, Z_{i}\right) \hat{g}^{\prime \top}\left(X_{i}^{\top} \beta\right) Z_{i} J_{\beta^{(1)}}^{\top} X_{i} Z_{i}^{\top} \mid X_{i}^{\top} \beta\right]\left\{\hat{E}\left[\hat{w}\left(X_{i}, Z_{i}\right) Z_{i} Z_{i}^{\top} \mid X_{i}^{\top} \beta\right]\right\}^{-1} Z_{i}\right\}=0, \\
& \text { (b) } n^{-1} \sum_{i=1}^{n}\left\{Y_{i}-Z_{i}^{\top} \hat{g}\left(X_{i}^{\top} \beta\right)\right\}\left\{\hat{g}^{\prime \top}\left(X_{i}^{\top} \beta\right) Z_{i} J_{\beta^{(1)}}^{\top} X_{i}\right. \\
& \left.-\hat{E}\left[\hat{g}^{\prime \top}\left(X_{i}^{\top} \beta\right) Z_{i} J_{\beta^{(1)}}^{\top} X_{i} Z_{i}^{\top} \mid X_{i}^{\top} \beta\right]\left\{\hat{E}\left[Z_{i} Z_{i}^{\top} \mid X_{i}^{\top} \beta\right]\right\}^{-1} Z_{i}\right\}=0, \\
& \text { (c) } n^{-1} \sum_{i=1}^{n}\left\{Y_{i}-Z_{i}^{\top} \hat{g}\left(X_{i}^{\top} \beta\right)\right\} \hat{w}\left(X_{i}, Z_{i}\right) \hat{g}^{\prime \top}\left(X_{i}^{\top} \beta\right) Z_{i} J_{\beta^{(1)}}^{\top} X_{i}=0 \text {, } \\
& \text { (d) } n^{-1} \sum_{i=1}^{n}\left\{Y_{i}-Z_{i}^{\top} \hat{g}\left(X_{i}^{\top} \beta\right)\right\} \hat{g}^{\top \top}\left(X_{i}^{\top} \beta\right) Z_{i} J_{\beta^{(1)}}^{\top} X_{i}=0 .
\end{aligned}
$$

The estimator defined by (a) is semiparametric efficient when the model is heteroscedastic, while the estimator defined by (b) is semiparametric efficient when the model is homoscedastic. Estimators defined by (c) and (d) correspond to two normal estimators. Table 1 summarizes the results. Moreover, we computed absolute errors (AE) for each functional component. $A E_{j}$ is defined as

$$
A E_{j}=\sum_{i=1}^{n}\left|\hat{g}_{j}\left(X_{i}^{\top} \hat{\beta}\right)-g_{j}\left(X_{i}^{\top} \beta_{0}\right)\right|, j=1,2,3
$$

Insert Table 1 about here

Several conclusions can be made from Table 1 . When the sample size $n$ increases, all four estimators (a)-(b) have smaller errors. Estimator (b) performs well for homoscedastic 
case, so does estimator (a) for heteroscedastic case. This finding agrees with semiparametric efficiency theory in Section 2.

\subsection{Real data analysis}

As an illustration, we apply the proposed method to a body fat data which is available at http:// lib.stat.cmu.edu/datasets/bodyfat. A variety of popular health books suggest that people could assess their health conditions by estimating their percentage of body fat. Unfortunately, it is inconvenient or costly nowadays to accurately measure body fat. The aim for analyzing this data set is to build a predictive model for percentage of body fat. To achieve this goal, we use the data set of 252 men with thirteen baseline predictors: AGE (age), BMI(body mass index ) and circumferences of various skinfold measurements [ NK(neck), CT(chest), AN(2 abdomen), HIP (hip), RAN (the ratio of 2 abdomen to hip), TN(thigh), KE(knee), AK(ankle), BS(biceps), FA(forearm) and WI(wrist)]. The response $(Y)$ is the logarithm of percentage of body fat. Due to the generating process of the response and possibly outliers, we delete a sample with $Y=0$, a sample with $Y=0.7$ and a sample with height $=29.5$ inches. The sample size is thus 249 .

Before applying our method, the predictors are transformed so that their marginal distribution are approximately $N(0,1)$. Moreover, the first 200 samples are used to fit the model, and then the rest 49 observations are utilized to evaluate the prediction ability of the underlying model. The prediction performance is measured by the absolute prediction error (APE), which is defined as $\left\{\left|y_{i}-\hat{y}_{i}\right|\right\}, i=1, \cdots, 49$. Consider the following homoscedastic model

$$
\begin{aligned}
Y= & g_{0}(U)+g_{1}(U) N K+g_{2}(U) C T+g_{3}(U) A N+g_{4}(U) H I P+g_{5}(U) T N \\
& +g_{6}(U) K E+g_{7}(U) A K+g_{8}(U) B S+g_{9}(U) F A+g_{10}(U) W I+\varepsilon,
\end{aligned}
$$

where $U=\beta_{1} A G E+\beta_{2} B M I+\beta_{3} R A N$ and $\varepsilon$ is the error term. Moreover, the variables AGE, BMI and RAN are transformed so that the marginal distributions are all $U[0,1]$.

Let $\beta=\left(\beta_{1}, \beta_{2}, \beta_{3}\right)^{\top}$. Based on the efficient estimating equation in Section 2, we get $\tilde{\beta}=(0.3372,0.9374,-0.0868)^{\top}$ and $R^{2}=0.8093$, where

$$
R^{2}=(S S T-S S E) / S S T, \quad S S T=\sum\left(Y_{i}-\operatorname{mean}\left(Y_{i}\right)\right)^{2}, \quad S S E=\sum\left(\tilde{Y}_{i}-Y_{i}\right)^{2}
$$


and $\tilde{Y}_{i}$ is the fitted value based on model (11). It is remarkable that the $R^{2}$ value implies (11) is a reasonable model to fit this real data. The median of APEs of the proposed model (11) is $0.3775(0.2713)$, where the values in the parentheses are MAD of APEs.

\section{Acknowledgment}

We sincerely thank the Editor, the AE, and two anonymous reviewers for their insightful comments and suggestions on the original version of the paper, which significantly improve the manuscript. Peng Lai's research was supported by National Natural Science Foundation of China (11301279) and Natural Science Foundation of Jiangsu Province for Youth (BK20140983). Qingzhao Zhang's research was supported by the China Postdoctoral Science Foundation (2014M550799) and the National Science Foundation of China (11401561). Qihua Wang's research was supported by the National Science Fund for Distinguished Young Scholars in China (10725106), the National Natural Science Foundation of China (11171331,11331011), a grant from the Key Lab of Random Complex Structure and Data Science, CAS and Natural Science Foundation of SZU.

\section{Appendix. Technical Proofs}

In order to study the proposed asymptotic properties, the following standard assumptions are imposed.

- C1. The density function $f(t)$ of $W^{\top} \eta$ is bounded away from zero and infinity on $\mathbf{T}_{\eta}=\left\{t: t=W^{\top} \eta, W \in \mathbf{A}_{W}, \eta \in \mathbf{B}_{n}^{\eta}=\left\{\eta:\|\eta\|=1,\left\|\eta-\eta_{0}\right\| \leq C n^{-1 / 2}\right\}\right\}, \mathbf{A}_{W}$ is the compact support of $W$. $f(t)$ has continuous derivatives up to order 2 on $\mathbf{T}_{\eta}$. Here $(W, \eta)$ can be $(X, \beta)$ and $(U, \gamma)$, respectively.

- $\mathrm{C} 2$. The coefficients $g_{j}(t), j=1, \ldots, q$, both have bounded and continuously derivatives up to order 2 on $\mathbf{T}_{\beta}$.

- C3. Assume $E\left(\varepsilon^{2} \mid X_{i}, Z_{i}\right)=\sigma^{2}\left(X_{i}, Z_{i}\right)=\varphi\left(U^{\top} \gamma_{0}\right), U=\left(X^{\top}, Z^{\top}\right)^{\top}$ and $\left\|\gamma_{0}\right\|=1$. $\varphi(\cdot)$ is bounded away from 0 and infinity, and has bounded derivative up to order 2 on $T_{\gamma}$. 
- C4. The functions $D_{1}(t)=E\left[h(X, Z) Z Z^{\top} \mid t\right]$ and $D_{2}(t)=E\left[h(X, Z) g^{\top \top}(t) Z J_{\beta_{0}^{(1)}}^{\top} X Z^{\top} \mid t\right]$, are twice continuously differentiable with bounded derivatives, where $h(X, Z)=$ $w(X, Z)$ or $1 . D_{1}(t)$ is nonsingular and $E\left(\|Z\|^{4} \mid t\right)$ is also bounded.

- C5. The kernel $K$ is a bounded and symmetric probability density function, satisfying the Lipschitz condition and

$$
\int_{-\infty}^{\infty} u^{2} K(u) d u \neq 0, \quad \int_{-\infty}^{\infty}|u|^{i} K(u) d u<\infty, \quad i=1,2, \ldots
$$

- C6. For any $s>2$, we have $E\left|Y_{i}\right|^{2 s}<\infty, E\left|Z_{j}\right|^{2 s}<\infty, j=1, \ldots, q, Z \in \mathbf{A}_{Z}$.

- C7. $n^{2 \epsilon-1} h_{v} \rightarrow \infty$ for some $\epsilon<1-s^{-1}, h_{v} \rightarrow 0, h_{v}=h, h_{1}$ or $h_{2} . n h_{1}^{4} \rightarrow 0, n h_{1}^{2} h^{4} \rightarrow$ $0, \frac{\log ^{2} n}{n h h_{1}^{3}} \rightarrow 0, \frac{\log ^{3} n}{n h h_{2}} \rightarrow 0, n h^{5}=O(1), n h_{2}^{5}=O(1)$.

- C8. The matrix $\Sigma_{1}=E\left[g^{\prime \top}\left(X^{\top} \beta_{0}\right) Z J_{\beta_{0}^{(1)}}^{\top} X-D_{2}\left(X^{\top} \beta_{0}\right) D_{1}^{-1}\left(X^{\top} \beta_{0}\right) Z\right]^{\otimes 2}$ is positive definite.

Remark 2 Condition C1 ensures that the denominators of the proposed nonparametric estimators are bounded away from 0 and infinity on $t \in \mathbf{T}_{\eta}$ for $\eta$ in a neighbour of $\eta_{0}$. Condition C2-C4 are some derivative conditions, they are standard smoothness conditions. Condition C5 is the usual assumptions for the kernel function. C6 and C7 are the necessary condition for the asymptotic normality or the uniform consistency of the estimators. Condition $C 8$ ensures that the asymptotic variance for the estimator $\beta_{0}$ exists.

Proof of Theorem 1. Let $p(\varepsilon, X, Z), p_{1}(\varepsilon \mid X, Z), p_{2}(X, Z)$ represent the density function of $(\varepsilon, X, Z)$, the conditional density function of $\varepsilon$ on $(X, Z)$ and the marginal density function of $(X, Z)$, respectively.

Note that

$$
p(\varepsilon, X, Z)=p_{1}(\varepsilon \mid X, Z) p_{2}(X, Z)
$$

Following Tsiatis (2006), we first consider the parametric submodels to derive the efficient score. We consider submodels $p_{1}\left(\varepsilon \mid X, Z, \theta_{1}\right), p_{2}\left(X, Z, \theta_{2}\right), g_{i}\left(X_{i}^{\top} \beta, \theta_{3 i}\right), i=1,2, \cdots, q$. Then the parameter of interest is $\beta^{(1)}$, the nuisance parameters are $\left\{\theta_{1}, \theta_{2}, \theta_{3 i}, i=1,2, \cdots, q\right\}$. 
Denote the score function for $\beta^{(1)}$ by $S_{\beta}$. From

$$
\begin{gathered}
\frac{\partial}{\partial \beta^{(1)}} \log p(\varepsilon, X, Z)=\frac{\partial}{\partial \beta^{(1)}} \log p_{1}(\varepsilon \mid X, Z)=\frac{p_{1, \varepsilon}^{\prime}(\varepsilon \mid X, Z)}{p_{1}(\varepsilon \mid X, Z)} \frac{\partial \varepsilon}{\partial \beta^{(1)}} \\
\text { and } \quad \frac{\partial \varepsilon}{\partial \beta^{(1)}}=\frac{\partial\left[Y-g^{\top}\left(X^{\top} \beta\right) Z\right]}{\partial \beta^{(1)}}=-g^{\prime \top}\left(X^{\top} \beta\right) Z J_{\beta^{(1)}}^{\top} X,
\end{gathered}
$$

we have

$$
S_{\beta}=-\frac{p_{1, \varepsilon}^{\prime}(\varepsilon \mid X, Z)}{p_{1}(\varepsilon \mid X, Z)} g^{\prime \top}\left(X^{\top} \beta\right) Z J_{\beta^{(1)}}^{\top} X .
$$

Denote the nuisance tangent space corresponding to $p_{1}, p_{2}$ and $g$ respectively $\Lambda_{1}, \Lambda_{2}$ and $\Lambda_{3}$. Thus we have

$$
\begin{aligned}
& \Lambda_{1}=\{S(\varepsilon, X, Z): E[\varepsilon S(\varepsilon, X, Z) \mid X, Z]=0, E[S(\varepsilon, X, Z) \mid X, Z]=0\}, \\
& \Lambda_{2}=\{S(X, Z): E[S(X, Z)]=0\}, \\
& \Lambda_{3}=\left\{S(\varepsilon, X, Z): E[S(\varepsilon, X, Z) \mid X, Z]=0, E[\varepsilon S(\varepsilon, X, Z) \mid X, Z]=h^{\top}\left(X^{\top} \beta\right) Z\right\},
\end{aligned}
$$

$h(\cdot)$ is a function dependent on $X^{\top} \beta$. Obviously, we find that $\Lambda_{1}, \Lambda_{2}$ and $\Lambda_{3}$ are mutually orthogonality. Hence the nuisance tangent space $\Lambda=\Lambda_{1} \oplus \Lambda_{2} \oplus \Lambda_{3}$, that is,

$$
\begin{aligned}
\Lambda= & \left\{f(Y, X, Z): E[f]=0, E[\varepsilon f \mid X, Z]=h^{\top}\left(X^{\top} \beta\right) Z,\right. \\
& \left.h\left(X^{\top} \beta\right) \text { is a function of } X^{\top} \beta\right\} .
\end{aligned}
$$

Note that $\Lambda^{\perp}$ is defined to be the orthogonal complement of $\Lambda$. In next lemma we derive the elements of $\Lambda^{\perp}$.

Lemma 1 For model (2), $\Lambda^{\perp}=\left\{\varepsilon f(X, Z): E\left[f(X, Z) Z^{\top} \mid X^{\top} \beta\right]=0\right\}$.

Proof: For any $h(Y, X, Z) \in \mathcal{H}, \mathcal{H}$ denotes the Hilbert space of $q$-dimensional meanzero finite-variance measurable functions (equipped with the covariance inner product), it suffices to show that $\varepsilon h_{1}(X, Z) \in \Lambda^{\perp}, h(Y, X, Z)-\varepsilon h_{1}(X, Z) \in \Lambda$ and this separation for $h(Y, X, Z)$ is unique. To ensure $h(Y, X, Z)-\varepsilon h_{1}(X, Z) \in \Lambda$, it must satisfy that $E\left[h(Y, X, Z)-\varepsilon h_{1}(X, Z)\right]=0$ and

$$
E\left[\varepsilon h(Y, X, Z)-\varepsilon^{2} h_{1}(X, Z) \mid X, Z\right]=h_{2}^{\top}\left(X^{\top} \beta\right) Z \text { for some } h_{2} \text {. }
$$


Thus

$$
h_{1}(X, Z)=\frac{1}{E\left[\varepsilon^{2} \mid X, Z\right]}\left\{E[\varepsilon h(Y, X, Z) \mid X, Z]-h_{2}^{\top}\left(X^{\top} \beta\right) Z\right\} .
$$

Since $\varepsilon h_{1}(X, Z) \in \Lambda^{\perp}, E\left[h_{1}(X, Z) Z^{\top} \mid X^{\top} \beta\right]=0$. Together with the above two equalities, we have

$$
\begin{gathered}
E\left\{\frac{1}{E\left[\varepsilon^{2} \mid X, Z\right]}\left[E[\varepsilon h(Y, X, Z) \mid X, Z] Z^{\top}-h_{2}^{\top}\left(X^{\top} \beta\right) Z Z^{\top}\right] \mid X^{\top} \beta\right\}=0 \\
\Rightarrow \quad h_{2}^{\top}\left(X^{\top} \beta\right)=E\left\{\frac{E[\varepsilon h(Y, X, Z) \mid X, Z] Z^{\top}}{E\left[\varepsilon^{2} \mid X, Z\right]} \mid X^{\top} \beta\right\}\left\{E\left[\frac{Z Z^{\top}}{E\left[\varepsilon^{2} \mid X, Z\right]} \mid X^{\top} \beta\right]\right\}^{-1} .
\end{gathered}
$$

Therefore any $h(Y, X, Z) \in \mathcal{H}$ can be separated into these two spaces uniquely. That is, $\Lambda^{\perp}=\left\{\varepsilon f(X, Z): E\left[f(X, Z) Z^{\top} \mid X^{\top} \beta\right]=0\right\}$.

Hereafter we focus to derive the efficient score function. Note that one score function of $\beta^{(1)}$ is $S_{\beta}=-\frac{p_{1, \varepsilon}^{\prime}(\varepsilon \mid X, Z)}{p_{1}(\varepsilon \mid X, Z)} g^{\top}\left(X^{\top} \beta\right) Z J_{\beta^{(1)}}^{\top} X$, then the efficient score can be defined as $S_{\beta}^{e f f}=S_{\beta}-\Pi\left(S_{\beta} \mid \Lambda\right)=\Pi\left(S_{\beta} \mid \Lambda^{\perp}\right)$.

By the proof of Lemma 1, we have $S_{\beta}^{e f f}(Y, X, Z)=\varepsilon h_{1}(X, Z)$, where

$$
\begin{aligned}
h_{1}(X, Z) & =\frac{1}{E\left[\varepsilon^{2} \mid X, Z\right]}\left[E\left[\varepsilon S_{\beta} \mid X, Z\right]-h_{2}^{\top}\left(X^{\top} \beta\right) Z\right], \\
h_{2}^{\top}\left(X^{\top} \beta\right) & =E\left\{\frac{E\left[\varepsilon S_{\beta} \mid X, Z\right] Z^{\top}}{E\left[\varepsilon^{2} \mid X, Z\right]} \mid X^{\top} \beta\right\}\left\{E\left[\frac{Z Z^{\top}}{E\left[\varepsilon^{2} \mid X, Z\right]} \mid X^{\top} \beta\right]\right\}^{-1} .
\end{aligned}
$$

Set $w(X, Z)=\frac{1}{E\left[\varepsilon^{2} \mid X, Z\right]}$. Note that $E_{p_{1}}\left\{-\frac{\left.p_{1, \varepsilon}^{\prime} \varepsilon \mid X, Z\right)}{p_{1}(\varepsilon \mid X, Z)} \varepsilon \mid X, Z\right\}=1$. For $h_{1}(X, Z)$, we have

$$
\begin{aligned}
h_{1}(X, Z)= & w(X, Z)\left\{g^{\prime \top}\left(X^{\top} \beta\right) Z J_{\beta^{(1)}}^{\top} X\right. \\
& \left.-E\left[w(X, Z) g^{\prime \top}\left(X^{\top} \beta\right) Z J_{\beta^{(1)}}^{\top} X Z^{\top} \mid X^{\top} \beta\right]\left\{E\left[w(X, Z) Z Z^{\top} \mid X^{\top} \beta\right]\right\}^{-1} Z\right\} .
\end{aligned}
$$

Therefore

$$
\begin{aligned}
S_{\beta_{0}}^{e f f}(Y, X, Z)= & \varepsilon w(X, Z)\left\{g^{\prime \top}\left(X^{\top} \beta_{0}\right) Z J_{\beta_{0}^{(1)}}^{\top} X\right. \\
& \left.-E\left[w(X, Z) g^{\prime \top}\left(X^{\top} \beta_{0}\right) Z J_{\beta_{0}^{(1)}}^{\top} X Z^{\top} \mid X^{\top} \beta_{0}\right]\left\{E\left[w(X, Z) Z Z^{\top} \mid X^{\top} \beta_{0}\right]\right\}^{-1} Z\right\} .
\end{aligned}
$$

Recall that $D_{1}\left(X_{i}^{\top} \beta\right)=E\left[w\left(X_{i}, Z_{i}\right) Z_{i} Z_{i}^{\top} \mid X_{i}^{\top} \beta\right]$ and

$$
D_{2}\left(X_{i}^{\top} \beta\right)=E\left[w\left(X_{i}, Z_{i}\right) g^{\prime T}\left(X_{i}^{\top} \beta\right) Z_{i} J_{\beta(1)}^{\top} X_{i} Z_{i}^{\top} \mid X_{i}^{\top} \beta\right] .
$$

Denote $U\left(X_{i}, Z_{i}, \beta\right)=g^{\prime T}\left(X_{i}^{\top} \beta\right) Z_{i} J_{\beta^{(1)}}^{\top} X_{i}-D_{2}\left(X_{i}^{\top} \beta\right) D_{1}^{-1}\left(X_{i}^{\top} \beta\right) Z_{i}$. Thus the efficient score function can be written as $S_{\text {eff }}=\varepsilon w(X, Z) U\left(X_{i}, Z_{i}, \beta\right)$.

To prove Theorem 2, we first give some lemmas. 
Lemma 2 Let $\left(T_{1}, Y_{1}\right), \ldots,\left(T_{n}, Y_{n}\right)$ be i.i.d. random vectors, where $Y_{i}$ s are scalar random variables. Assume further that $E\left|Y_{1}\right|^{s}<\infty$ and $\sup _{t} \int|y|^{s} f(t, y) d y<\infty$, where $f$ denotes the joint density of $\left(T_{1}, Y_{1}\right)$. Let $K(\cdot)$ be a bounded positive function with a bounded support, satisfying the Lipschitz condition. Then

$$
\sup _{t \in \mathbf{T}}\left|\frac{1}{n} \sum_{i=1}^{n}\left[K_{h}\left(T_{i}-t\right) Y_{i}-E\left(K_{h}\left(T_{i}-t\right) Y_{i}\right)\right]\right|=O_{p}\left\{\left(\frac{\log n}{n h}\right)^{1 / 2}\right\},
$$

provided $0<h \rightarrow 0$ and $n^{2 \epsilon-1} h \rightarrow \infty$ for some $\epsilon<1-s^{-1}, s>2$, where $h$ is a bandwidth and $\mathbf{T}$ is some closed set.

The proof of the Lemma can be found in Mack and Silverman (1982).

Lemma 3 Suppose that conditions in Theorem 2 hold. Then

$$
\begin{aligned}
& \sup _{t \in \mathbf{T}, \beta \in \mathbf{B}_{n}}\|\hat{g}(t ; \beta)-g(t)\|=O_{p}\left(\left\{\frac{\log n}{n h}\right\}^{1 / 2}+h^{2}\right), \\
& \sup _{t \in \mathbf{T}, \beta \in \mathbf{B}_{n}}\left\|\hat{g}^{\prime}(t ; \beta)-g^{\prime}(t)\right\|=O_{p}\left(\left\{\frac{\log n}{n h^{3}}\right\}^{1 / 2}+h\right) .
\end{aligned}
$$

This lemma is proved in Theorem 1 of Xue and Wang (2012).

Lemma 4 Suppose that conditions in Theorem 2 hold. Then for any $\beta \in \mathbf{B}_{n}$,

$$
\frac{1}{\sqrt{n}} \sum_{i=1}^{n}\left(g\left(X_{i}^{\top} \beta\right)-\hat{g}\left(X_{i}^{T} \beta\right)\right)^{T} Z_{i} w\left(X_{i}, Z_{i}\right) S_{e i}(\beta)=o_{p}(1)
$$

where $S_{e i}(\beta)=g^{\prime \top}\left(X_{i}^{\top} \beta\right) Z_{i} J_{\beta^{(1)}}^{\top} X_{i}-D_{2}\left(X_{i}^{\top} \beta\right) D_{1}^{-1}\left(X_{i}^{\top} \beta\right) Z_{i}$.

Proof. From (7), and by Lemma 2, we have $\frac{1}{n} \sum_{i=1}^{n} Z_{i} Z_{i}^{\top} K_{h}\left(t_{i}-t\right)-f(t) E\left(Z Z^{\top} \mid t\right)=$ $O_{p}\left(h^{2}+\left[\frac{\log n}{n h}\right]^{1 / 2}\right)$, let $\Sigma_{11}(t)=f(t) E\left(Z Z^{\top} \mid t\right), t=X^{\top} \beta$, thus,

$$
\begin{aligned}
\hat{g}(t)-g(t)= & \Sigma_{11}^{-1}(t) \frac{1}{n} \sum_{i=1}^{n} Z_{i} \varepsilon_{i} K_{h}\left(t_{i}-t\right)+\Sigma_{11}^{-1}(t) \frac{1}{n} \sum_{i=1}^{n} Z_{i} Z_{i}^{\top} K_{h}\left(t_{i}-t\right) h g^{\prime}(t)\left(\frac{t_{i}-t}{h}\right) \\
& +\Sigma_{11}^{-1}(t) \frac{1}{n} \sum_{i=1}^{n} Z_{i} Z_{i}^{\top} K_{h}\left(t_{i}-t\right) \frac{h^{2}}{2} g^{\prime \prime}(t)\left(\frac{t_{i}-t}{h}\right)^{2}+O_{p}\left(h^{4}\right) .
\end{aligned}
$$


Therefore,

$$
\begin{aligned}
& \frac{1}{\sqrt{n}} \sum_{i=1}^{n}\left(g\left(X_{i}^{\top} \beta\right)-\hat{g}\left(X_{i}^{T} \beta\right)\right)^{T} Z_{i} w\left(X_{i}, Z_{i}\right) S_{e i}(\beta) \\
& =o_{p}(1)-\frac{1}{\sqrt{n}} \sum_{i=1}^{n} \varepsilon_{i}\left\{\frac{1}{n} \sum_{j=1}^{n} w\left(X_{j}, Z_{j}\right) S_{e j}(\beta) Z_{j}^{\top} K_{h}\left(t_{j}-t_{i}\right)\right\} \Sigma_{11}^{-1}(t) Z_{i} \\
& \quad+\frac{1}{\sqrt{n}} \sum_{i=1}^{n}\left\{\frac{1}{n} \sum_{j=1}^{n} w\left(X_{j}, Z_{j}\right) S_{e j}(\beta) Z_{j}^{\top} K_{h}\left(t_{j}-t_{i}\right)\left[h g^{\prime}\left(t_{j}\right) \frac{t_{j}-t_{i}}{h}-\frac{h^{2}}{2} g^{\prime \prime}\left(t_{j}\right)\left(\frac{t_{j}-t_{i}}{h}\right)^{2}\right]\right\} Z_{i} Z_{i}^{\top} \\
& :=o_{p}(1)+E_{a 1}+E_{a 2} .
\end{aligned}
$$

Since $E\left(w\left(X_{j}, Z_{j}\right) S_{e j}(\beta) Z_{j}^{\top} K_{h}\left(t_{j}-t_{i}\right)\right)=0$, we can get $E\left(E_{a 1}^{\otimes 2}\right)=O\left(\frac{1}{n h}\right)$, thus $E_{a 1}=$ $O_{p}\left(\frac{1}{\sqrt{n h}}\right)=o_{p}(1)$. Similarly, $E_{a 2}=O_{p}\left(\sqrt{h^{2}}\right)=o_{p}(1)$. Lemma 4 is then proved.

Proof of Theorem 2. Denote $S_{e i}(\beta)=g^{\prime \top}\left(X_{i}^{\top} \beta\right) Z_{i} J_{\beta^{(1)}}^{\top} X-D_{2}\left(X_{i}^{\top} \beta\right) D_{1}^{-1}\left(X_{i}^{\top} \beta\right) Z$. Now we focus on the following estimating equation

$$
Q_{n}(\hat{\beta}) \doteq \frac{1}{\sqrt{n}} \sum_{i=1}^{n}\left(Y_{i}-\hat{g}^{\top}\left(X_{i}^{\top} \hat{\beta}\right) Z_{i}\right) \hat{w}\left(X_{i}, Z_{i}\right) \hat{S}_{e i}(\hat{\beta})=0
$$

where $\hat{S}_{e i}(\hat{\beta})=\hat{g}^{\prime \top}\left(X_{i}^{\top} \hat{\beta}\right) Z_{i} J_{\hat{\beta}^{(1)}}^{\top} X_{i}-\hat{D}_{2}\left(X_{i}^{\top} \hat{\beta}\right) \hat{D}_{1}^{-1}\left(X_{i}^{\top} \hat{\beta}\right) Z$. It is remarkable that $Q_{n}(\beta)=$ 0 is the sample version of $n^{-1 / 2} \sum_{i=1}^{n} S_{\beta}^{e f f}\left(Y_{i}, X_{i}, Z_{i}\right)=0$ with some 'plug-in' estimators.

Firstly, it is easy to see that

$$
\begin{aligned}
Q_{n}(\hat{\beta}) & =\frac{1}{\sqrt{n}} \sum_{i=1}^{n}\left(g\left(X_{i}^{T} \beta_{0}\right)-\hat{g}\left(X_{i}^{T} \hat{\beta}\right)\right)^{T} Z_{i} \hat{w}\left(X_{i}, Z_{i}\right) \hat{S}_{e i}(\hat{\beta})+\frac{1}{\sqrt{n}} \sum_{i=1}^{n} \varepsilon_{i} \hat{w}\left(X_{i}, Z_{i}\right) \hat{S}_{e i}(\hat{\beta}) \\
& :=E_{1}+E_{2} .
\end{aligned}
$$

For $E_{1}$, we have

$$
\begin{aligned}
E_{1}= & \frac{1}{\sqrt{n}} \sum_{i=1}^{n}\left(g\left(X_{i}^{\top} \beta_{0}\right)-\hat{g}\left(X_{i}^{\top} \hat{\beta}\right)\right)^{\top} Z_{i} w\left(X_{i}, Z_{i}\right) S_{e i}\left(\beta_{0}\right) \\
& +\frac{1}{\sqrt{n}} \sum_{i=1}^{n}\left(g\left(X_{i}^{\top} \beta_{0}\right)-\hat{g}\left(X_{i}^{\top} \hat{\beta}\right)\right)^{\top} Z_{i}\left[\hat{w}\left(X_{i}, Z_{i}\right)-w\left(X_{i}, Z_{i}\right)\right] S_{e i}\left(\beta_{0}\right) \\
& +\frac{1}{\sqrt{n}} \sum_{i=1}^{n}\left(g\left(X_{i}^{\top} \beta_{0}\right)-\hat{g}\left(X_{i}^{\top} \hat{\beta}\right)\right)^{\top} Z_{i} w\left(X_{i}, Z_{i}\right)\left[\hat{S}_{e i}(\hat{\beta})-S_{e i}\left(\beta_{0}\right)\right] \\
& +\frac{1}{\sqrt{n}} \sum_{i=1}^{n}\left(g\left(X_{i}^{\top} \beta_{0}\right)-\hat{g}\left(X_{i}^{\top} \hat{\beta}\right)\right)^{\top} Z_{i}\left[\hat{w}\left(X_{i}, Z_{i}\right)-w\left(X_{i}, Z_{i}\right)\right]\left[\hat{S}_{e i}(\hat{\beta})-S_{e i}\left(\beta_{0}\right)\right] \\
:= & E_{11}+E_{12}+E_{13}+E_{14} .
\end{aligned}
$$


For $E_{11}$, we have

$$
\begin{aligned}
E_{11}= & \frac{1}{\sqrt{n}} \sum_{i=1}^{n}\left(g\left(X_{i}^{\top} \beta_{0}\right)-g\left(X_{i}^{\top} \hat{\beta}\right)\right)^{\top} Z_{i} w\left(X_{i}, Z_{i}\right) S_{e i}\left(\beta_{0}\right) \\
& +\frac{1}{\sqrt{n}} \sum_{i=1}^{n}\left(g\left(X_{i}^{\top} \hat{\beta}\right)-\hat{g}\left(X_{i}^{\top} \hat{\beta}\right)\right)^{\top} Z_{i} w\left(X_{i}, Z_{i}\right) S_{e i}\left(\beta_{0}\right) \\
= & E_{111}+E_{112} .
\end{aligned}
$$

By Lemma $4, E_{112}=o_{p}(1)$, and we have

$$
E_{11}=-\frac{1}{\sqrt{n}} \sum_{i=1}^{n} S_{e i}\left(\beta_{0}\right) w\left(X_{i}, Z_{i}\right) Z_{i}^{\top} g^{\prime}\left(X_{i}^{\top} \beta_{0}\right) X_{i}^{\top} J_{\beta_{0}^{(1)}}\left(\hat{\beta}^{(1)}-\beta_{0}^{(1)}\right)+o_{p}(1) .
$$

For $E_{12}$, note the definitions of the initial estimators of $\hat{g}_{I}(\cdot)$ and $\hat{\beta}_{I}$, by Lemma 2-3, and the assumption for $\sigma^{2}(\cdot)$, similar to the proof of Lemma 4 in Ma and Zhu (2013), it follows

$$
\sup _{X \in \mathbf{A}_{X}, Z \in \mathbf{A}_{Z}}\left\|\hat{\sigma}^{2}(X, Z)-\sigma^{2}(X, Z)\right\|=O_{p}\left(h_{2}^{2}+\frac{\log n}{\sqrt{n h_{2}}}\right) .
$$

Therefore, note that $\hat{w}(\cdot)=\frac{1}{\hat{\sigma}^{2}(\cdot)}$, by Lemma $3,(17)$ and condition $\mathrm{C} 7$, we get

$$
E_{12}=\frac{1}{\sqrt{n}} \sum_{i=1}^{n}\left(g\left(X_{i}^{\top} \beta_{0}\right)-\hat{g}\left(X_{i}^{\top} \hat{\beta}\right)\right)^{\top} Z_{i}\left[\hat{w}\left(X_{i}, Z_{i}\right)-w\left(X_{i}, Z_{i}\right)\right] S_{e i}\left(\beta_{0}\right)=o_{p}(1) .
$$

Similarly, we can prove $E_{13}=o_{p}(1)$ and $E_{14}=o_{p}(1)$. Thus

$$
E_{1}=-\frac{1}{\sqrt{n}} \sum_{i=1}^{n} S_{e i}\left(\beta_{0}\right) w\left(X_{i}, Z_{i}\right) Z_{i}^{\top} g^{\prime}\left(X_{i}^{\top} \beta_{0}\right) J_{\beta_{0}^{(1)}}^{\top} X_{i}\left(\hat{\beta}^{(1)}-\beta_{0}^{(1)}\right)+o_{p}(1) .
$$

For the term $E_{2}$, we have

$$
\begin{aligned}
E_{2}= & \frac{1}{\sqrt{n}} \sum_{i=1}^{n} \varepsilon_{i} w\left(X_{i}, Z_{i}\right) S_{e i}\left(\beta_{0}\right)+\frac{1}{\sqrt{n}} \sum_{i=1}^{n} \varepsilon_{i}\left[\hat{w}\left(X_{i}, Z_{i}\right)-w\left(X_{i}, Z_{i}\right)\right] S_{e i}\left(\beta_{0}\right) \\
& +\frac{1}{\sqrt{n}} \sum_{i=1}^{n} \varepsilon_{i}\left[\hat{w}\left(X_{i}, Z_{i}\right)-w\left(X_{i}, Z_{i}\right)\right]\left[\hat{S}_{e i}(\hat{\beta})-S_{e i}\left(\beta_{0}\right)\right] \\
& +\frac{1}{\sqrt{n}} \sum_{i=1}^{n} \varepsilon_{i} w\left(X_{i}, Z_{i}\right)\left[\hat{S}_{e i}(\hat{\beta})-S_{e i}\left(\beta_{0}\right)\right] \\
:= & E_{21}+E_{22}+E_{23}+E_{24} .
\end{aligned}
$$

Similarly, it can be obtained

$$
E_{2}=\frac{1}{\sqrt{n}} \sum_{i=1}^{n} \varepsilon_{i} w\left(X_{i}, Z_{i}\right) S_{e i}\left(\beta_{0}\right)+o_{p}(1)
$$


By (13),(18) and (20), it follows

$$
\begin{aligned}
& \frac{1}{\sqrt{n}} \sum_{i=1}^{n} S_{e i}\left(\beta_{0}\right) w\left(X_{i}, Z_{i}\right) Z_{i}^{\top} g^{\prime}\left(X_{i}^{\top} \beta_{0}\right) X_{i}^{\top} J_{\beta_{0}^{(1)}}\left(\hat{\beta}^{(1)}-\beta_{0}^{(1)}\right) \\
= & \frac{1}{\sqrt{n}} \sum_{i=1}^{n} \varepsilon_{i} w\left(X_{i}, Z_{i}\right) S_{e i}\left(\beta_{0}\right)+o_{p}(1) .
\end{aligned}
$$

Therefore,

$$
\begin{aligned}
\sqrt{n}\left(\hat{\beta}^{(1)}-\beta_{0}^{(1)}\right)= & {\left[\frac{1}{n} \sum_{i=1}^{n} w\left(X_{i}, Z_{i}\right) S_{e i}\left(\beta_{0}\right) Z_{i}^{\top} g^{\prime}\left(X_{i}^{\top} \beta_{0}\right) X_{i}^{\top} J_{\beta_{0}^{(1)}}\right]^{-1} } \\
& \times \frac{1}{\sqrt{n}} \sum_{i=1}^{n} \varepsilon_{i} w\left(X_{i}, Z_{i}\right) S_{e i}\left(\beta_{0}\right)+o_{p}(1) .
\end{aligned}
$$

Denote the items of the right side of (22) as $A_{n}^{-1} W_{n}+o_{p}(1)$. Clearly, $A_{n} \rightarrow_{p} \Sigma_{1}^{*}$ and $W_{n} \rightarrow_{d} N\left(0, \Sigma_{1}\right)$, where

$$
\begin{aligned}
\Sigma_{1}^{*} & =E\left[w(X, Z) S_{e}\left(\beta_{0}\right) Z^{\top}\left(g^{\prime}\left(X^{\top} \beta_{0}\right)\right) X^{\top} J_{\beta_{0}^{(1)}}\right] \\
\text { and } \quad \Sigma_{1} & =E\left[w(X, Z) S_{e}\left(\beta_{0}\right) S_{e}^{\top}\left(\beta_{0}\right)\right] .
\end{aligned}
$$

It is noted that $\Sigma_{1}^{*}=\Sigma_{1}=E\left[S_{\beta}^{e f f} S_{\beta}^{\text {eff }}\right]$. Thus we have

$$
\sqrt{n}\left(\hat{\beta}^{(1)}-\beta_{0}^{(1)}\right) \stackrel{\mathcal{D}}{\longrightarrow} N\left(0, \Sigma_{1}^{-1}\right) .
$$

\section{References}

Carroll, R., Fan, J., Gijbels, I., and Wand, M. (1997), "Generalized partially linear singleindex models," Journal of the American Statistical Association, 92, 477-489.

Fan, J. and Gijbels, I. (1996), Local polynomial modelling and its applications, vol. 66, Chapman \& Hall/CRC.

Feng, S. and Xue, L. (2013), "Variable selection for single-index varying-coefficient model," Frontiers of Mathematics in China, 8, 541-565.

- (2015), "Model detection and estimation for single-index varying coefficient model," Journal of Multivariate Analysis, 139, 227-244. 
Huang, Z. and Zhang, R. (2013), "Profile empirical-likelihood inferences for the singleindex-coefficient regression model," Statistics and Computing, 23, 455-465.

Lai, P. and Wang, Q. (2014), "Semiparametric efficient estimation for partially linear single-index models with responses missing at random," Journal of Multivariate Analysis, 128, 33-50.

Lai, P., Wang, Q., and Zhou, X.-H. (2014), "Variable selection and semiparametric efficient estimation for the heteroscedastic partially linear single-index model," Computational Statistics \& Data Analysis, 70, 241-256.

Ma, Y. and Zhu, L. (2013), "Doubly robust and efficient estimators for heteroscedastic partially linear single-index model allowing high-dimensional covariates," Journal of the Royal Statistical Society: Series B (Statistical Methodology), 75, 305-322.

Mack, Y. and Silverman, B. (1982), "Weak and strong uniform consistency of kernel regression estimates," Probability Theory and Related Fields, 61, 405-415.

Tsiatis, A. (2006), Semiparametric theory and missing data, Springer Verlag.

Xue, L. and Pang, Z. (2013), "Statistical inference for a single-index varying-coefficient model," Statistics and Computing, 23, 589-599.

Xue, L. and Wang, Q. (2012), "Empirical likelihood for single-index varying-coefficient models," Bernoulli, 18, 836-856.

Yu, Y. and Ruppert, D. (2002), "Penalized spline estimation for partially linear singleindex models," Journal of the American Statistical Association, 97, 1042-1054.

Zhu, L. and Xue, L. (2006), "Empirical likelihood confidence regions in a partially linear single-index model," Journal of the Royal Statistical Society: Series B (Statistical Methodology), 68, 549-570. 
Table 1: Summary of the simulation

\begin{tabular}{|c|c|c|c|c|c|c|}
\hline \multirow[t]{2}{*}{$\mathrm{n}$} & \multirow[t]{2}{*}{ estimator } & \multicolumn{2}{|r|}{$\left(\hat{\beta}_{2}-\beta_{2}\right)^{2}$} & $A E_{1}$ & $A E_{2}$ & $A E_{3}$ \\
\hline & & Mean $\times 10^{4}\left(S D \times 10^{4}\right)$ & Mean $\times 10^{4}\left(S D \times 10^{4}\right)$ & Median(MAD) & Median(MAD) & Median(MAD) \\
\hline & & & $e_{i} \sim N(0,1)$ & & & \\
\hline \multirow[t]{4}{*}{100} & (a) & $0.9593(1.4096)$ & $3.7750(5.4123)$ & $0.1745(0.0314)$ & $0.1327(0.0303)$ & $0.1440(0.0341)$ \\
\hline & (b) & $0.9141(1.3398)$ & $3.5936(5.1763)$ & $0.1740(0.0313)$ & $0.1320(0.0303)$ & $0.1442(0.0339)$ \\
\hline & (c) & $1.0473(1.6003)$ & $4.2421(6.5839)$ & $0.1759(0.0317)$ & $0.1326(0.0305)$ & $0.1449(0.0341)$ \\
\hline & (d) & $0.9938(1.5342)$ & $4.0086(6.2901)$ & $0.1759(0.0315)$ & $0.1321(0.0304)$ & $0.1444(0.0340)$ \\
\hline \multirow[t]{5}{*}{200} & (a) & $0.4179(0.6400)$ & $1.6527(2.4636)$ & $0.1387(0.0222)$ & $0.0873(0.0194)$ & $0.0952(0.0207)$ \\
\hline & (b) & $0.3963(0.5852)$ & $1.5691(2.2745)$ & $0.1378(0.0221)$ & $0.0872(0.0194)$ & $0.0952(0.0207)$ \\
\hline & (c) & $0.4574(0.6692)$ & $1.1847(2.7189)$ & $0.1386(0.0222)$ & $0.0873(0.0194)$ & $0.0951(0.0207)$ \\
\hline & (d) & $0.4326(0.6112)$ & $1.7488(2.5006)$ & $0.1390(0.0221)$ & $0.0872(0.0194)$ & $0.0954(0.0206)$ \\
\hline & & & $\sim N\left(0, \exp \left(U_{i}^{\top} \gamma_{0} / 2\right)\right)$ & & & \\
\hline \multirow[t]{4}{*}{100} & (a) & $1.1502(1.7886)$ & $4.5352(6.7866)$ & $0.2104(0.0363)$ & $0.1436(0.0340)$ & $0.1605(0.0415)$ \\
\hline & (b) & $1.1982(1.8526)$ & $4.6741(6.9417)$ & $0.2112(0.0362)$ & $0.1439(0.0338)$ & $0.1597(0.0415)$ \\
\hline & (c) & $1.3483(2.2517)$ & $5.4827(9.3104)$ & $0.2135(0.0365)$ & $0.1444(0.0342)$ & $0.1599(0.0417)$ \\
\hline & (d) & $1.3302(2.1566)$ & $5.3800(8.7749)$ & $0.2134(0.0362)$ & $0.1447(0.0339)$ & $0.1600(0.0416)$ \\
\hline \multirow[t]{4}{*}{200} & (a) & $0.4616(0.7070)$ & $1.8465(2.7567)$ & $0.1466(0.0252)$ & $0.1032(0.0235)$ & $0.1118(0.0237)$ \\
\hline & (b) & $0.5211(0.7759)$ & $2.0614(3.0027)$ & $0.1467(0.0250)$ & $0.1035(0.0235)$ & $0.1125(0.0238)$ \\
\hline & (c) & $0.5509(0.8374)$ & $2.2366(3.4693)$ & $0.1464(0.0253)$ & $0.1037(0.0235)$ & $0.1119(0.0237)$ \\
\hline & (d) & $0.5564(0.7901)$ & $2.2499(3.2306)$ & $0.1466(0.0252)$ & $0.1040(0.0235)$ & $0.1125(0.0237)$ \\
\hline
\end{tabular}

